Service social

\title{
The Woman Client-Providing Human Services in a Changing World, par D.S. Burden et N. Gottlieb (éds.), New York, Tavistock Publication, 1987.
}

\section{Geneviève Martin}

Volume 37, numéro 1-2, 1988

Par-delà les barrières des sexes

URI : https://id.erudit.org/iderudit/706398ar

DOI : https://doi.org/10.7202/706398ar

Aller au sommaire du numéro

Éditeur(s)

École de service social de l'Université Laval

ISSN

1708-1734 (numérique)

Découvrir la revue

Citer ce compte rendu

Martin, G. (1988). Compte rendu de [The Woman Client - Providing Human Services in a Changing World, par D.S. Burden et N. Gottlieb (éds.), New York, Tavistock Publication, 1987.] Service social, 37(1-2), 271-272.

https://doi.org/10.7202/706398ar d'utilisation que vous pouvez consulter en ligne.

https://apropos.erudit.org/fr/usagers/politique-dutilisation/ 
mais tout au moins pouvons-nous croire en un volume représentant une étape importante dans le renouvellement des études sur la condition masculine; celles-ci apporteront une contribution, d'une part distincte des travaux féministes des deux dernières décades mais, d'autre part, complémentaires à ceux-ci.

Jocelyn LINDSAY

École de service social,

Université Laval.

The Woman Client - Providing Human Services in a Changing World, par D.S. Burden et N. Gottueb (éds.), New York, Tavistock Publication, 1987.

Ce volume se situe dans la continuité de l'ouvrage de E. Norman et A. Mancuso (Women's Issues and Social Work Practice, Itasca (III.), Peacock, 1980), première production traitant spécifiquement de la femme et du service social. Le texte vise à fournir un ancrage pour l'amélioration de l'enseignement, de la recherche et de l'intervention concernant les femmes. Il est proposé de tenir compte des changements survenus dans les aspects privés et politiques de leur vie. Les résultats de recherches empiriques portant sur les problématiques vécues par elles doivent également inspirer l'enseignement, la recherche et la pratique. D'autres disciplines intervenant auprès des femmes peuvent aussi faire grand usage de ce livre.

Il faut mentionner au départ que le volume est écrit par un groupe d'auteures. L'abondance des thèmes abordés est compensée par la clarté de la division en quatre parties (éléments génériques, intervention, problèmes et services, types de population), par la précision de la présentation de chacune des parties et par la rigueur de la synthèse qu'offre chacun des chapitres. Sans être toujours identiques, les rubriques utilisées permettent rapidement de se situer. Incidemment, la cueillette de toutes les recommandations pour l'enseignement pourrait constituer un curriculum féministe. Les références bibliographiques complétant chacun des chapitres sont très pertinentes et généralement récentes.

En introduction, on trouve une définition du sexisme :

"Le sexisme est un système non fonctionnel par lequel on utilise des stéréotypes liés au sexe; il tend à déprécier les femmes et à faire de la discrimination contre elles. Ce n'est pas quelque chose qui est fait aux femmes par les hommes, mais plutôt un système affectant à la fois hommes et femmes, le système d'éducation et la littérature. Le sexisme fait partie d'un système culturel dans lequel on perçoit les hommes comme ayant plus de valeur que les femmes." (P.2)

Le service social est interpellé quant au sexisme. Les auteures examinent les effets de ce phénomène sur la vie des clientes et des intervenantes, le rôle du sexisme dans les interventions, sa présence dans les organisations, le sexisme en relation avec la santé mentale des hommes et des femmes et le sexisme comme système politique. 
Il est impossible de reprendre toutes les idées de ce volume. Toutefois, soulignons quelques questions importantes. En quoi la recherche féministe (chap. 6) peut-elle être stratégie de changement pour les femmes? Il est proposé que les résultats de recherches documentant les conditions sociales des femmes puissent être utilisés pour chercher des alternatives à ces conditions. On suggère que les femmes participent à la formulation de problèmes de recherche portant sur des aspects non étudiés de leur vie.

Quant à l'intervention, on préconise (chap. 8) qu'elle soit basée sur une évaluation tenant compte des valeurs féministes et de l'analyse du discours des femmes sur les aspects multidimensionnels de leur environnement et sur leurs facteurs de stress.

Dans l'enseignement de l'administration des services sociaux, on suggère de faire davantage place à l'autonomie des femmes, à l'action positive en leur faveur et à leur cheminement de carrière. Au niveau du champ d'intervention de la santé, on rappelle l'ampleur des efforts du Mouvement de santé des femmes pour rehausser le statut des professionnelles de la santé, pour améliorer l'autonomie des femmes face à leur corps et à leur santé et pour démystifier le pouvoir médical (chap. 12). Le chapitre 15 aborde la situation de la femme incarcérée, et celle de l'intervenante dans le milieu de la justice. Malgré le contexte américain dans lequel se situe clairement l'exposé, on reconnaît les enjeux présents dans notre milieu et le peu de cas qu'on a fait jusqu'ici de la pauvreté vécue par les femmes aux prises avec la justice, de leur monoparentalité, de leur isolement, de leur piètre condition de santé et d'éducation.

L'adolescence vue du point de vue des femmes, tel est l'objet du chapitre 19. C'est fort pertinent de lire sur « les dilemmes sociaux de la dernière décennie : la sexualité des adolescents, les risques de santé durant l'adolescence et la violence envers les adolescentes".

Enfin, le dernier chapitre est consacré au statut social des femmes âgées et aux suggestions précises pour cette clientèle souvent ignorée.

L'ensemble du volume présente un aspect didactique pouvant devenir assez lourd pour la lecture. L'effort nécessaire est très valable car tout se passe comme si les synthèses dans chacun des chapitres pouvaient servir d'assises pour de nouvelles analyses et interventions sociales tenant définitivement compte de la spécificité des femmes. C'est un outil indispensable pour se préparer à intervenir auprès de cette clientèle et pour élaborer des programmes sociaux en faveur des femmes.

Geneviève Martin

École de service social, Université Laval. 